\title{
SDSS J2222+2745: A GRAVITATIONALLY LENSED SEXTUPLE QUASAR WITH A MAXIMUM IMAGE SEPARATION OF 15".1 DISCOVERED IN THE SLOAN GIANT ARCS SURVEY*
}

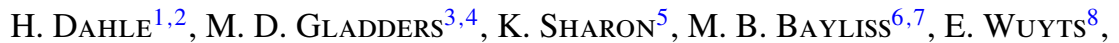 \\ L. E. Abramson ${ }^{3,4}$, B. P. Koester ${ }^{9}$, N. Groeneboom ${ }^{1}$, T. E. BrincKmann ${ }^{10}$, M. T. Kristensen ${ }^{10}$, \\ M. O. Lindholmer ${ }^{10}$, A. Nielsen ${ }^{10}$, J.-K. Krogager ${ }^{10}$, AND J. P. U. Fynbo ${ }^{10}$ \\ ${ }^{1}$ Institute of Theoretical Astrophysics, University of Oslo, P.O. Box 1029, Blindern, NO-0315 Oslo, Norway; hdahle@ astro.uio.no \\ ${ }^{2}$ Centre of Mathematics for Applications, University of Oslo, Blindern, NO-0315 Oslo, Norway \\ ${ }^{3}$ Department of Astronomy \& Astrophysics, The University of Chicago, 5640 S. Ellis Avenue, Chicago, IL 60637, USA \\ ${ }^{4}$ Kavli Institute for Cosmological Physics at the University of Chicago, 5640 South Ellis Avenue, Chicago, IL 60637, USA \\ ${ }^{5}$ Department of Astronomy, University of Michigan, 500 Church Street, Ann Arbor, MI 48109, USA \\ ${ }^{6}$ Harvard-Smithsonian Center for Astrophysics, 60 Garden Street, Cambridge, MA 02138, USA \\ ${ }^{7}$ Department of Physics, Harvard University, 17 Oxford Street, Cambridge, MA 02138, USA \\ ${ }^{8}$ Max-Planck-Institut für extraterrestrische Physik, Giessenbackstrasse 1, D-85748 Garching bei München, Germany \\ ${ }^{9}$ Department of Physics, University of Michigan, 450 Church Street, Ann Arbor, MI 48109, USA \\ ${ }^{10}$ Dark Cosmology Centre, Niels Bohr Institute, Juliane Maries Vej 30, DK-2100 Copenhagen O, Denmark \\ Received 2012 November 3; accepted 2013 May 18; published 2013 August 5
}

\begin{abstract}
We report the discovery of a unique gravitational lens system, SDSS J2222+2745, producing five spectroscopically confirmed images of a $z_{s}=2.82$ quasar lensed by a foreground galaxy cluster at $z_{l}=0.49$. We also present photometric and spectroscopic evidence for a sixth lensed image of the same quasar. The maximum separation between the quasar images is $15^{\prime \prime}$. 1 . Both the large image separations and the high image multiplicity are in themselves rare among known lensed quasars, and observing the combination of these two factors is an exceptionally unlikely occurrence in present data sets. This is only the third known case of a quasar lensed by a cluster, and the only one with six images. The lens system was discovered in the course of the Sloan Giant Arcs Survey, in which we identify candidate lenses in the Sloan Digital Sky Survey and target these for follow-up and verification with the $2.56 \mathrm{~m}$ Nordic Optical Telescope. Multi-band photometry obtained over multiple epochs from 2011 September to 2012 September reveals significant variability at the $\sim 10 \%-30 \%$ level in some of the quasar images, indicating that measurements of the relative time delay between quasar images will be feasible. In this lens system, we also identify a bright $(g=21.5)$ giant arc corresponding to a strongly lensed background galaxy at $z_{s}=2.30$. We fit parametric models of the lens system, constrained by the redshift and positions of the quasar images and the redshift and position of the giant arc. The predicted time delays between different pairs of quasar images range from $\sim 100$ days to $\sim 6 \mathrm{yr}$.
\end{abstract}

Key words: galaxies: clusters: general - gravitational lensing: strong - quasars: individual (SDSS J2222+2745)

Online-only material: color figures

\section{INTRODUCTION}

Strongly gravitationally lensed quasars with image separations $\gtrsim 10^{\prime \prime}$ are exceptionally rare. Such large image splittings require a lensing mass corresponding to a galaxy cluster, and a fortuitous close line-of-sight alignment of such a mass distribution and a background quasar. In fact, only two such systems have previously been discovered, SDSS J1004+4112 (Inada et al. 2003) with a maximum image separation of $14 . .6$ and SDSS J1029+2623 (Inada et al. 2006) with a maximum image separation of 22".5. Such systems provide unique information on the abundances and internal structure of galaxy clusters, yielding stringent tests of the current $\Lambda \mathrm{CDM}$ paradigm, both through their observed abundances (e.g., Hennawi et al. 2007), the relative fractions of different image multiplicities (e.g., Oguri \& Keeton 2004), and from detailed models of the cluster mass distribution based on the available constraints (e.g., Sharon et al. 2005; Oguri 2010; Oguri et al. 2013), including measured time delays between the quasar images (Fohlmeister et al. 2008,

\footnotetext{
* Based on observations made with the Nordic Optical Telescope, operated on the island of La Palma jointly by Denmark, Finland, Iceland, Norway, and Sweden, in the Spanish Observatorio del Roque de los Muchachos of the Instituto de Astrofisica de Canarias.
}

2013). Being effectively point sources, the positions of multiply lensed quasar images provide mass modeling constraints which are free from the ambiguities that sometimes plague the identification of multiple images of strongly lensed galaxies, particularly the fainter demagnified images located closest to the mass center.

As shown by Refsdal (1964), the relative time delays of multiple quasar images visible around the lensing mass center can provide an estimate of the Hubble parameter $H_{0}$. However, as $H_{0}$ is presently increasingly well constrained by a host of methods, including gravitational lensing time delays from galaxy-scale lenses, the main interest of quasar time delays is related to their ability to provide unique detailed constraints on the slope of the projected matter density distribution over the range of radii where the quasar images are found (e.g., Refsdal 2004).

In this paper, we present the discovery of a third lensing system where a quasar is being multiply lensed by a galaxy cluster, but with a higher image multiplicity than the previously known such systems. The discussion in subsequent sections of this paper describes the evidence for the lensing interpretation of this system and the identification of an increasing number of components in the lensing system, through analysis of 
Table 1

Log of NOT/MOSCA Imaging Observations

\begin{tabular}{lccc}
\hline \hline Epoch & Filter & Exposure Time & FWHM \\
\hline 2011 Sep 24.93 UT & $g$ & $2 \times 300 \mathrm{~s}$ & $0^{\prime \prime} 87$ \\
2011 Sep 24.94 UT & $r$ & $2 \times 150 \mathrm{~s}$ & $0^{\prime} .81$ \\
2011 Sep 24.95 UT & $i$ & $2 \times 150 \mathrm{~s}$ & $0^{\prime} \cdot 74$ \\
\hline 2012 Sep 13.97 UT & $u$ & $3 \times 600 \mathrm{~s}$ & $0^{\prime \prime} 81$ \\
2012 Sep 12.96 UT & $g$ & $3 \times 600 \mathrm{~s}$ & 0.70 \\
2012 Sep 13.04 UT & $r$ & $3 \times 300 \mathrm{~s}$ & $0^{\prime \prime} 68$ \\
2012 Sep 12.95 UT & $i$ & $3 \times 300 \mathrm{~s}$ & $0^{\prime \prime} 61$ \\
\hline 2012 Sep 15.98 UT & $g$ & $3 \times 600 \mathrm{~s}$ & $0^{\prime \prime} 68$ \\
\hline
\end{tabular}

progressively deeper photometric and spectroscopic data, coupled with parametric modeling of the lens.

In Section 2, we describe the initial identification of SDSS J2222+2745 as a likely gravitational lens system in the course of a larger survey for strong cluster lenses. The spectroscopic confirmation of the three brightest lensed images of the quasar is outlined in Section 3.1, and Section 3.2 details the evidence for three additional fainter quasar images from deeper imaging of the field, coupled with further spectroscopic observations. In Section 3.3, we report photometry of the quasar images, some showing significant brightness changes over a time span of 1 yr. In Section 3.4, we provide a summary of the observed properties of the lensing cluster, and in Section 4 we compute a parametric model of the lens system, which is used to predict time delays. Our main results are summarized in Section 5. Unless otherwise noted, throughout this work we assume an $\Omega_{m}=0.3, \Omega_{\Lambda}=0.7$, and $H_{0}=70 \mathrm{~km} \mathrm{~s}^{-1}$ cosmology.

\section{LENS CANDIDATE SELECTION AND INITIAL IMAGING}

We have undertaken a search for cluster lenses, the Sloan Giant Arcs Survey (Hennawi et al. 2008; Bayliss et al. 2011; M. D. Gladders et al. 2013, in preparation), based on public data from the Sloan Digital Sky Survey (SDSS; York et al. 2000). Potential lines of sight for lensing by clusters are identified by running an automated cluster-finding algorithm on the SDSS imaging data. This is followed by visual inspection, by multiple persons in our collaboration, of color images of these lines of sight, generated from the SDSS data. New lens candidates are flagged and given a grade according to the likelihood of being a true lens, and a final grade is calculated for each lens candidate. The list of lens candidates is then checked against public data bases such as the NASA/IPAC Extragalactic Database, and the remaining candidates after the removal of known lenses are targeted for deeper follow-up imaging. In 2011 September, we targeted such lens candidates selected from the SDSS Data Release 8 (Aihara et al. 2011) using the MOsaic CAmera (MOSCA) at the $2.56 \mathrm{~m}$ Nordic Optical Telescope (NOT). MOSCA is a $2 \times 2$ mosaic of four $2048 \times 2048$ Loral-Lesser thinned CCDs with excellent blue sensitivity (quantum efficiency $>90 \%$ across the SDSS $g$ band), mounted at the Cassegrain focus of the telescope. MOSCA is normally used in $2 \times 2$ binned mode, yielding a pixel scale of 0.217 pixel $^{-1}$ and a total field of view of $7.7 \times 7.7$.

SDSS J2222+2745 was imaged using MOSCA in 2011 September (see Table 1 for details) with our nominal survey exposure time of $2 \times 300 \mathrm{~s}$ in the $g$ band. Based on a visual inspection of the raw data, it was noted as a probable lens, and subject to immediate follow-up with $2 \times 150 \mathrm{~s}$ exposures in each of the SDSS $r$ and $i$ bands. In addition to the apparent blue

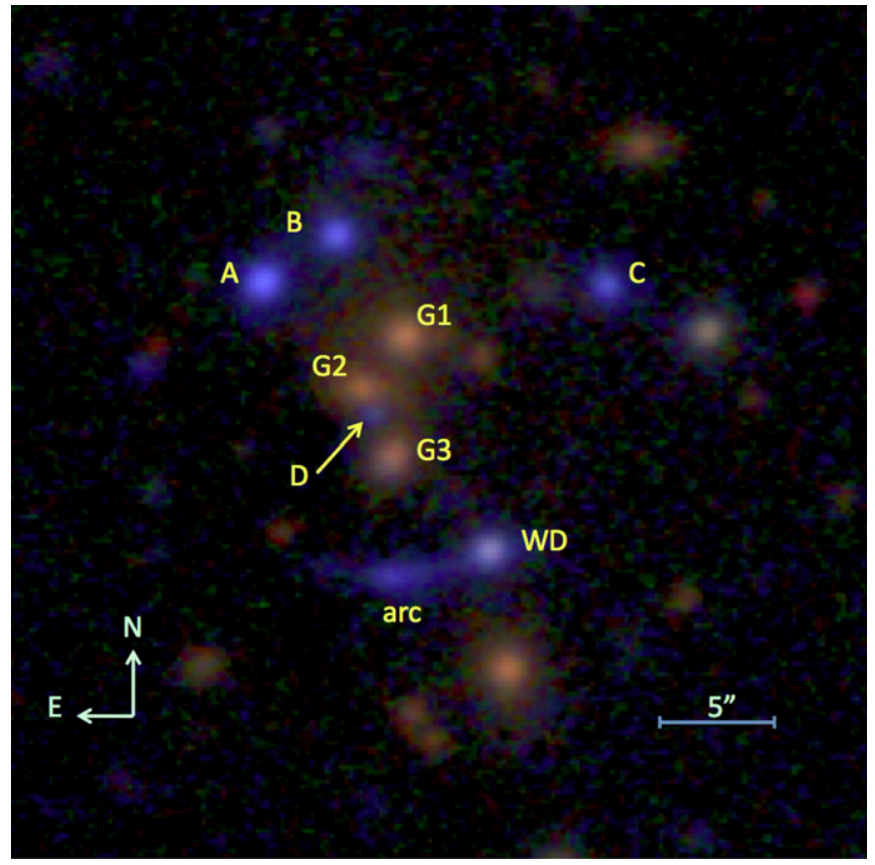

Figure 1. NOT/MOSCA gri color composite image of SDSS J2222+2745 from our initial epoch of photometry (see Table 1). The four brightest quasar images are labeled A-D, the three bright foreground cluster galaxies are labeled G1-G3, and a foreground white dwarf is labeled WD.

(A color version of this figure is available in the online journal.)

arc south of the cluster core, three blue stellar images (labeled "A," "B," and "C" in Figure 1) located at similar clustercentric radii as the arc, were considered as candidate multiple images of a gravitationally lensed quasar. In addition, three red galaxies, labeled "G1-G3" in Figure 1, were seen in the cluster core. Publicly available photometric redshift estimates from SDSS (see Table 2) indicated that all three galaxies are consistent with being located in a cluster at $z \sim 0.50$.

The stellar source (labeled "WD" in Figure 1) of magnitude $g=21.09$, similar in apparent brightness to A and B, seen superposed on the giant arc, was also noted as a candidate quasar image. Although this image has a significantly redder color $(g-i=1.33$ ) than $\mathrm{A}$ and $\mathrm{B}$, it might be considerably reddened by extinction in galaxies (the galaxy corresponding to the arc being one potential such candidate) along its line of sight, and it was kept as a target for future spectroscopic follow-up.

\section{FOLLOW-UP OBSERVATIONS AND RESULTS}

\subsection{Spectroscopic Confirmation of the Three Brightest Quasar Images}

The spectroscopic observations described in this paper were obtained using the Andalucia Faint Object Spectrograph and Camera (ALFOSC) at the NOT. We used grism 4, covering a wavelength range $3800-9000 \AA$ with a resolution of about $R=350$ and dispersion $3.0 \AA$ pixel $^{-1}$. The ALFOSC pixels were binned by a factor of two in the dispersion direction but kept unbinned at the native pixel scale of $0{ }^{\prime} .188$ pixel $^{-1}$ in the spatial direction. The slit width was $1^{\prime \prime}$. 3 . Redward of $\sim 7000 \AA$ strong fringing is present in the spectra, effectively restricting the upper limit of the spectral range to this value unless special care is taken during the observations to suppress the effects of fringing. An order-sorter filter was used to suppress secondorder contamination in the spectra. 
Table 2

SDSS DR8 Astrometry, Photometry, and Photometric Redshift Estimates for the

Three Brightest Quasar Images and Three Brightest Galaxies in SDSS J2222+2745

\begin{tabular}{|c|c|c|c|c|c|c|c|c|}
\hline Object & $\begin{array}{l}\text { R.A. } \\
\text { (h m s) }\end{array}$ & $\begin{array}{l}\text { Decl. } \\
\text { (d m s) }\end{array}$ & $\begin{array}{c}u \\
\text { SDSS }\end{array}$ & $\begin{array}{c}g \\
\text { SDSS }\end{array}$ & $\begin{array}{c}r \\
\text { SDSS }\end{array}$ & $\begin{array}{c}i \\
\text { SDSS }\end{array}$ & $\begin{array}{c}z \\
\text { SDSS }\end{array}$ & $\begin{array}{c}\text { Photometric } \\
\text { Redshift }\end{array}$ \\
\hline A & $22: 22: 09.04$ & $+27: 45: 37.9$ & $24.55 \pm 0.93$ & $21.10 \pm 0.03$ & $20.67 \pm 0.04$ & $20.87 \pm 0.07$ & $21.00 \pm 0.27$ & $\ldots$ \\
\hline $\mathrm{C}$ & $22: 22: 07.90$ & $+27: 45: 37.8$ & $24.50 \pm 0.93$ & $21.51 \pm 0.05$ & $21.14 \pm 0.05$ & $21.28 \pm 0.10$ & $22.14 \pm 0.63$ & $\ldots$ \\
\hline G1 & $22: 22: 08.57$ & $+27: 45: 35.6$ & $25.42 \pm 2.41$ & $20.92 \pm 0.08$ & $19.00 \pm 0.03$ & $18.05 \pm 0.02$ & $17.65 \pm 0.05$ & $0.485 \pm 0.015$ \\
\hline G2 & 22:22:08.70 & $+27: 45: 33.0$ & $24.33 \pm 1.01$ & $22.13 \pm 0.08$ & $20.86 \pm 0.05$ & $19.88 \pm 0.04$ & $19.33 \pm 0.07$ & $0.528 \pm 0.157$ \\
\hline
\end{tabular}

Notes. Epoch: 2009 October 18.19 UT. The SDSS imaging is too shallow to provide data for the three fainter quasar images D, E, and F.

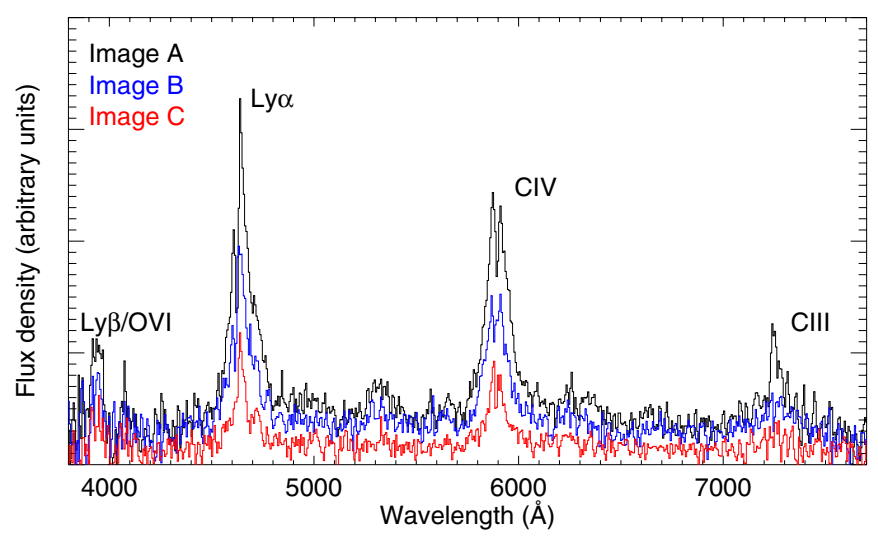

Figure 2. NOT/ALFOSC spectra of the three brightest quasar images A-C. The most prominent quasar emission lines, redshifted to $z=2.82$, are indicated in the figure.

(A color version of this figure is available in the online journal.)

On 2012 August 21-22, a series of ALFOSC spectra were obtained in the area of SDSS J2222+2745: three $1200 \mathrm{~s}$ spectra were obtained with the rotation angle set to provide simultaneous coverage of the $\mathrm{A}$ and $\mathrm{B}$ images. In addition, two single spectra of $1000 \mathrm{~s}$ exposure time were obtained to simultaneously cover image $\mathrm{C}$ and galaxy $\mathrm{G} 1$ and image $\mathrm{B}$ and image $\mathrm{C}$, respectively. Finally, the blue giant arc and the stellar source superposed on it (labeled "WD" in Figure 1) were observed for a total exposure time $3 \times 1000 \mathrm{~s}$. The spectra were wavelength calibrated using $\mathrm{He}+\mathrm{Ne}$ arc lamps and flux calibrated based on observations of the spectrophotometric standard star BD+33 2642. As shown in Figure 2, the three images $\mathrm{A}, \mathrm{B}$, and $\mathrm{C}$ all have identical spectra modulo noise, containing typical spectral features of a quasar (e.g., Vanden Berk et al. 2001) with a set of prominent emission lines, redshifted to $z=2.82$. This demonstrates that the lensing interpretation is correct for these three quasar images.

Despite having a significantly redder color, the stellar source superposed on the giant arc was also targeted for ALFOSC spectroscopy as a potential additional image of the quasar. Although a significant continuum was detected, emission lines were completely absent at the wavelengths of the prominent quasar emission lines in the $\mathrm{A}, \mathrm{B}$, and $\mathrm{C}$ component spectra. A more careful analysis of the spectrum reveals the typical signatures of a DZ white dwarf (WD; e.g., Koester et al. 2011).

\subsection{Identification of the $D, E$, and $F$ Images}

Having confirmed the three images A-C as gravitationally lensed images of the same quasar, two alternative image configurations may be considered: SDSS J2222+2745 is either a naked-cusp lens with three quasar images, similar to SDSS J1029+2623 (Oguri et al. 2008) or a five-image system similar to SDSS J1004+4112 (Inada et al. 2008). Being able to distinguish between the two cases is important, not only for the understanding of the SDSS J2222+2745 lens system itself, but also in the context of using the statistics of cluster-lensed quasars as a cosmological tool. Naked cusps, i.e., lensing configurations where the source lies on a tangential caustic that extends outside a radial caustic, are extremely rare among galaxy-scale lenses, the only known candidate among $\sim 100$ such lens systems being APM 08279+5255 (Lewis et al. 2002). However, due to their shallower central matter density slopes, naked-cusp lenses are expected to be much more common for clusters than for galaxies. Oguri \& Keeton (2004) predicted that 30\%-60\% of all cluster lenses will be of this type, the fraction depending on the central slope and triaxiality of clusters.

To further investigate the lensing system configuration of SDSS J2222+2745, we searched our initial MOSCA imaging for potential stellar sources with blue colors consistent with the quasar. A blue source, labeled " $D$ " in Figure 1, was identified just south of, and partially superposed on, galaxy G2. The source was apparently stellar, but the modest exposure time and overlap with G2 made this conclusion somewhat uncertain. A set of deeper ugri images in better seeing was obtained at the NOT in 2012 September (see Table 1). These images revealed image D to have a clearly stellar point-spread function (PSF), motivating further spectroscopic follow-up of this image. On 2012 September 16-17 UT, a series of $8 \times 2400 \mathrm{~s}$ integrations were made using ALFOSC, using the same setup as for the earlier observations described in Section 3.1. The telescope pointing was dithered along the slit between exposures to improve fringe suppression at the red end of the spectrum. The spectra were flux calibrated based on observations of the spectrophotometric standard star $\mathrm{BD}+332642$.

The slit orientation is illustrated in Figure 3, providing simultaneous spectroscopic coverage of images B and D, galaxies G2 and G3, and the giant arc. Inspection of the reduced twodimensional spectrum (see Figure 4) reveals the strong spectral line in the quasar B image around $4640 \AA$, corresponding to Ly $\alpha$ at the redshift of the quasar. An $\operatorname{Ly} \alpha$ line is also clearly evident at this wavelength in the spectrum of the D image, confirming its identification as a gravitationally lensed image of the quasar. More surprisingly, an Ly $\alpha$ line at the same redshift is also seen superposed on the spectrum of galaxy G3, providing clear indication of a fifth (E) quasar image spatially coincident with this galaxy. The extracted one-dimensional spectra of images D and $\mathrm{E}$ in $\mathrm{a} \sim 1200 \AA$ wide region surrounding the Ly $\alpha$ line of the quasar are plotted in Figure 5: a clear peak is seen at the location of the Ly $\alpha$ line in both the D and E image spectra. Redward of 


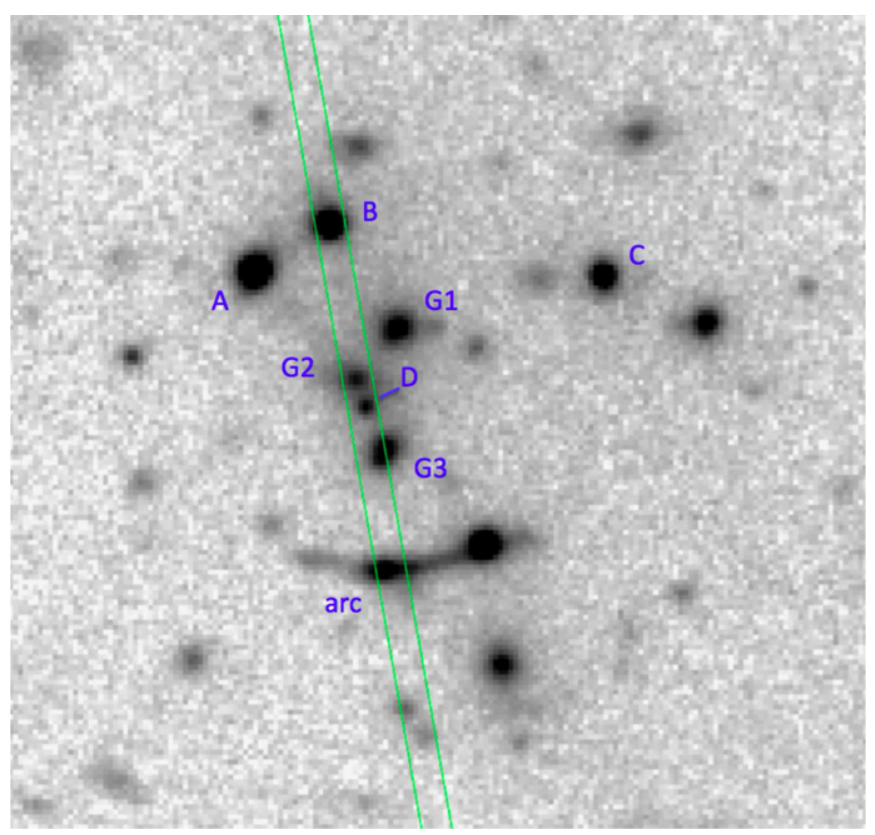

Figure 3. Position of the slit for spectroscopic observations of SDSS J2222+2745 using ALFOSC in 2012 September. The field of view and orientation are the same as for Figure 1. The background image is a $1 \mathrm{~h} g$-band MOSCA image combining all exposures obtained in this passband in 2012 September (see Table 1).

(A color version of this figure is available in the online journal.)

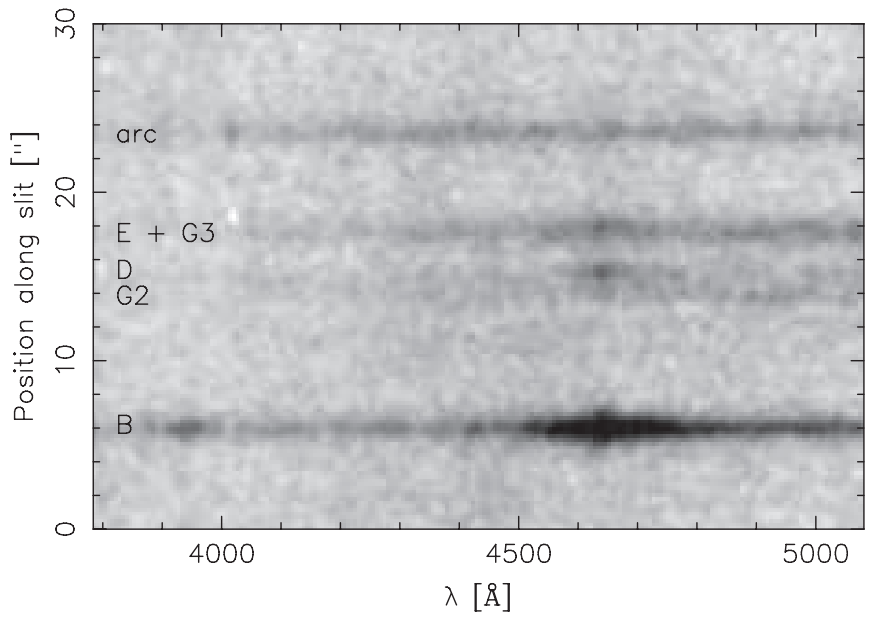

Figure 4. Reduced two-dimensional spectrum with slit orientation as shown in Figure 3. The brightest spectrum near the bottom is the B image of the quasar. The locations of other sources along the slit are indicated along the left margin. The D and E images (the latter superposed on the spectrum of galaxy G3) display clear Ly $\alpha$ emission lines at the same redshift as the B image. The image has been Gaussian smoothed on a scale of FWHM $=12 \AA$ in the spectral direction and $\mathrm{FWHM}=0 . \prime 4$ in the spatial direction.

this line, there is some visible discrepancy in the slopes of the two spectra. This discrepancy is caused by the spatial superposition of image $\mathrm{E}$ and the red galaxy G3, the latter having a continuum which is rising toward longer wavelengths.

Figure 6 shows a scaled subtraction of the second-epoch $i$-band image from the $g$-band image (see Table 1). The PSF of the $i$-band image was matched to that of the $g$-band image by convolving it with an elliptical Gaussian kernel. The $i$-band image was then scaled so that the subtraction optimally removes the light of galaxies G1-G3. Any remaining flux, shown in the negative in Figure 6, is bluer than galaxies G1-G3, and should be considered candidate images of the quasar.

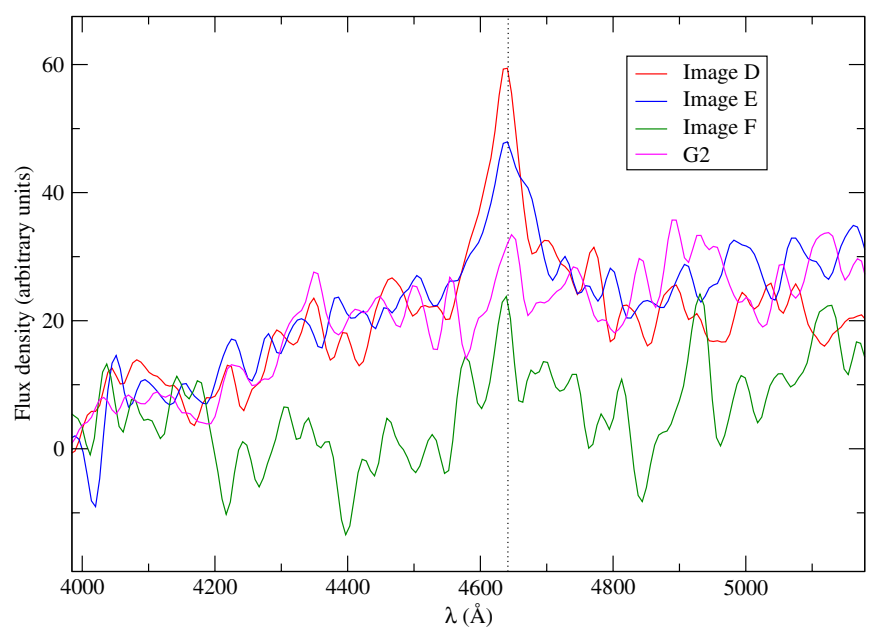

Figure 5. Spectra of the D, E, and F images and galaxy G2, showing the spectral region surrounding the $\operatorname{Ly} \alpha$ emission line. The wavelength of Ly $\alpha$ at the $z=2.82$ redshift of the quasar is indicated by the vertical dotted line. To dampen small-scale noise the spectra have been smoothed with a Gaussian filter of $\mathrm{FWHM}=18 \AA$.

(A color version of this figure is available in the online journal.)

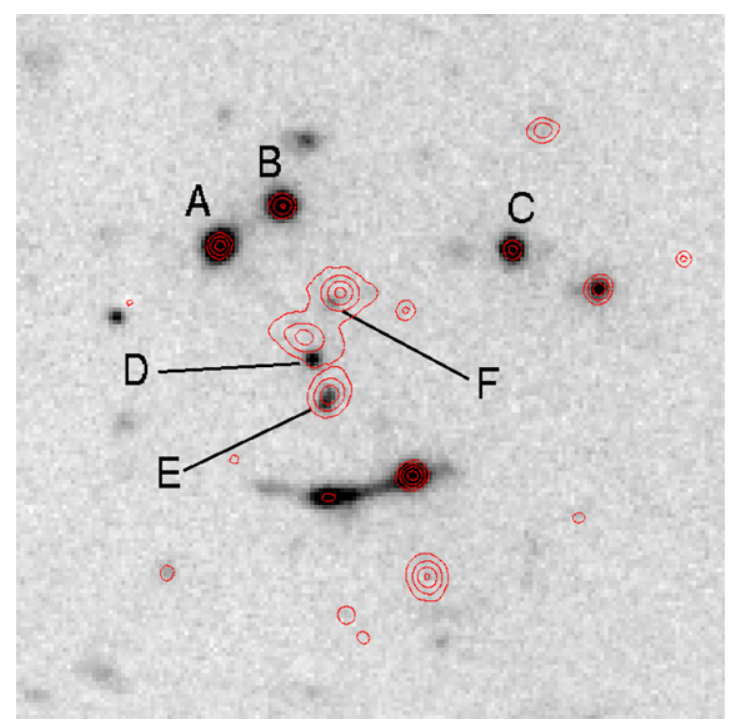

Figure 6. Residuals after subtracting the second-epoch stacked $i$-band image from the $g$-band image. Prior to the subtraction, the $i$-band image was PSFmatched by convolution with an elliptical Gaussian kernel and then scaled to match the $g$-band flux of galaxies G1-G3. The field of view and orientation are the same as for Figure 1. To indicate the locations of the galaxies we also show intensity contours from the $i$-band image. Objects with excess blue light in the cluster core are candidate quasar images. Images A-E are clearly confirmed by spectroscopy and readily visible in this image subtraction; a possible sixth quasar image $(\mathrm{F})$ is also indicated.

(A color version of this figure is available in the online journal.)

In Figure 6, image D is obvious. A comparison of this residual image to the residual quasar images $\mathrm{A}-\mathrm{C}$ shows that it has a PSF consistent with a point source, as expected. Additionally, there is excess blue flux at the spectroscopically indicated position of image E; this flux is obviously extended, however. Figure 7, which shows the $u$-band image with contours from the $g-i$ subtraction overlaid, shows the reason for this; the excess blue light is a combination of light from the core of galaxy G3, and offset from this, excess blue light attributable to image $\mathrm{E}$.

Finally, in Figure 6 there is also an additional blue excess source, near galaxy G1, which we label F in Figures 6 and 7. 


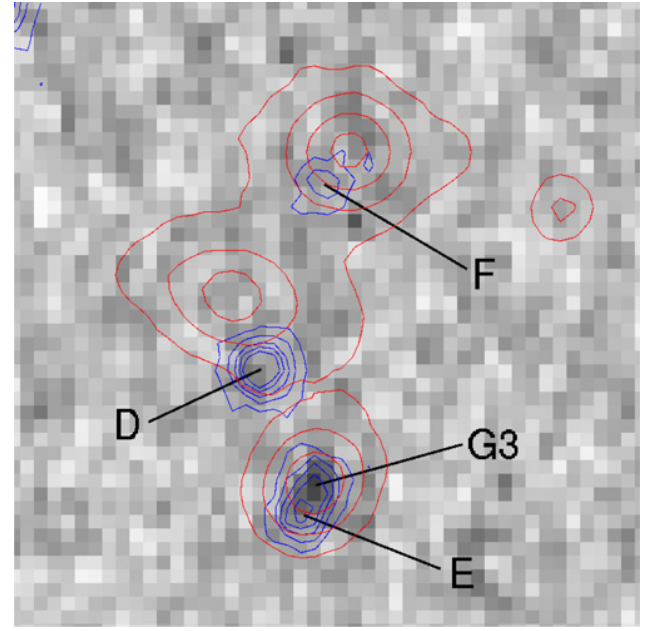

Figure 7. Zoomed view-four times finer than Figure 6-of the $u$-band image. Red contours, as in Figure 6, show the second-epoch $i$-band image. Blue contours show the residual $g-i$ shown in gray scale in Figure 6. Note that at the position of image $\mathrm{E}$, the residual blue light is extended, and that it partially coincides with an excess of $u$-band light that is most prominent at the center of galaxy G3. The peak of the excess blue light in $g-i$ is offset from the center of G3; we interpreted this light as the sum of an image of the quasar (E) and a blue core in galaxy G3.

(A color version of this figure is available in the online journal.)

This source is consistent with being a point source. One of the slit placements from the initial spectroscopic observations includes this possible quasar image, and Figure 5 includes a spectrum extracted at that location. The extracted spectrum suggests the presence of Ly $\alpha$ emission; taken together, the imaging and spectroscopic data suggest that this source is a sixth image of the quasar.

\subsection{Photometry of Quasar Images}

To achieve the most accurate photometry of images of the quasar, we have constructed a model of the light distribution in the cluster core region using GALFIT (Peng et al. 2010). The input PSF for each of the models discussed below was in all cases constructed by fitting bright, isolated and unsaturated stars using a multi-component Moffat profile using GALFIT. Typically three Moffat components were used; we have explored the effects of PSF reference star selection and find that it introduces an uncertainty of less than $0.01 \mathrm{mag}$ for the brighter quasar images, with no significant biases.

The GALFIT model of the light distribution was initially constructed using a stack of the second-epoch $g, r$, and $i$ images. Sources were initially placed at locations of obvious galaxies, and at the six quasar image positions discussed above. Quasar images are PSF components, and all galaxies are Sérsic profile components. We continued adding components to the model iteratively at the locations of any significant residuals revealed by the modeling process, until a final complete model incorporating all significant sources was achieved. This model was then translated to individual epoch and filter images by transforming the image coordinates to match the image to be analyzed, fixing those coordinates, and then refitting the model component magnitudes, sizes, and shapes to the image to be analyzed.

The modeling process described above reveals that the images of the quasar include a contribution from the underlying host galaxy, as is seen in the two other quasars strongly lensed by galaxy clusters (Inada et al. 2005; Oguri et al. 2013). The data show that the host galaxy is blue-unlike the red hosts seen in
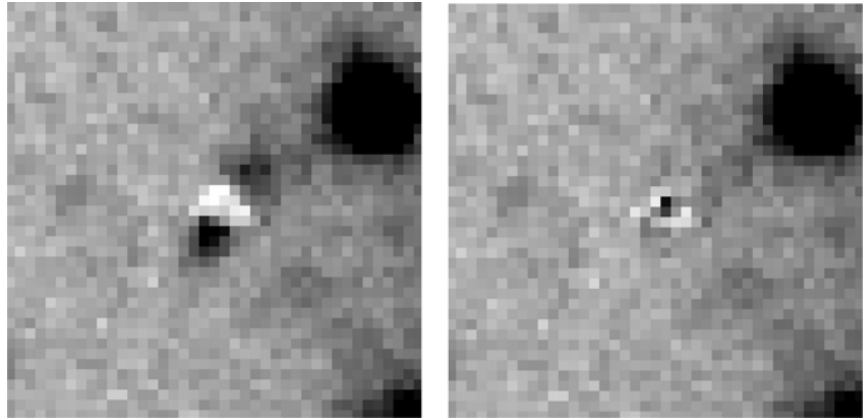

Figure 8. Left panel: the residuals in a $40 \times 40$ pixel region from the $g$-band image resulting from a single PSF GALFIT model centered on image A. Right panel: the same region fit by a PSF+Sérsic profile. Note that the PSF-only fit clearly indicates the presence of an underlying host galaxy component, and that it is oriented tangentially to the cluster center, as expected, and that this component is well fit by a single Sérsic profile.

the other two quasar-cluster lenses - and is most clearly seen in the brightest (and thus most likely the most magnified) image A. Figure 8 shows residuals from the full GALFIT model around image A; only the components for image A have been removed to specifically highlight residuals at that location. Two model variants are shown-one in which only a PSF component is used, and a second model for A consisting of a PSF component plus a single Sérsic component; this second model is a much better fit at that location.

In the $g$ band, including the host galaxy makes the fitted quasar 0.086 mag fainter. In our judgment, none of the other images of the quasar allow for clear modeling of the host galaxy component-image B is bright enough, but complicated by a second galaxy less than 2 arcsec to the northwest, and images $\mathrm{C}-\mathrm{F}$ are insufficiently magnified. Thus, for images $\mathrm{B}-\mathrm{D}$, in order to measure the apparent magnitude of each quasar image as robustly as possible, we proceed by measuring the quasar brightness using a simple PSF-only fit in GALFIT, and then correct each measured magnitude fainter by $0.086 \mathrm{mag}-$ i.e., by the fractional host galaxy contribution as measured from image A. This presumes that the quasar and host galaxy magnifications at each image location are the same; providing the host is compact and not too close to a caustic this assumption is reasonable and this supposition is well supported by the lack of giant arc images associated with the quasar host. This approach also in principle presumes no temporal variation in the quasar intrinsic brightness, since such variations would produce variation in the fractional correction. While not formally true (see below), that effect is second order and insignificant; the simple procedure we follow here for correcting for host galaxy light avoids systematic noise that would be introduced by attempting to model the host galaxy independently in multiple images. For reference, in addition to the $g$ band above, the correction for the host galaxy in $r$ and $i$ is measured to be 0.084 and $0.071 \mathrm{mag}$, respectively. Within the uncertainties, this correction is the same across bands-i.e., the host galaxy has a color comparable to the quasar. For the $u$ band, the image is of insufficient depth to model the host galaxy directly, we adopt the same correction as the $g$ band. The resulting photometry for images A-D, across four bands and two epochs, is reported in Table 3. Uncertainties are calculated by using the full GALFIT model to generate realizations of each measured frame with appropriate noise, which are then refit. The measured scatter in the photometry of quasar images, combined in quadrature with the estimated calibration uncertainty, is reported. For the 
Table 3

Photometry of QSO Images

\begin{tabular}{lcccccc}
\hline \hline Data Frame & \multicolumn{5}{c}{ Quasar Image } \\
\cline { 2 - 7 } & $\mathrm{A}$ & $\mathrm{B}$ & $\mathrm{C}$ & $\mathrm{D}$ & $\mathrm{E}$ & $\mathrm{F}$ \\
\hline $\boldsymbol{g}$-band first epoch & $21.03 \pm 0.01$ & $21.52 \pm 0.01$ & $22.13 \pm 0.01$ & $23.48 \pm 0.04$ & $24.32 \pm 0.09$ & $25.21 \pm 0.24$ \\
$g$-band second epoch & $21.15 \pm 0.01$ & $21.57 \pm 0.01$ & $22.22 \pm 0.01$ & $23.74 \pm 0.03$ & $24.22 \pm 0.08$ & $24.81 \pm 0.07$ \\
\hline$r$-band first epoch & $20.66 \pm 0.01$ & $21.13 \pm 0.01$ & $21.69 \pm 0.01$ & $23.13 \pm 0.11$ & $23.92 \pm 0.09$ & $24.81 \pm 0.24$ \\
$r$-band second epoch & $20.80 \pm 0.01$ & $21.16 \pm 0.01$ & $21.79 \pm 0.01$ & $23.46 \pm 0.08$ & $23.82 \pm 0.08$ & $24.41 \pm 0.07$ \\
\hline$i$-band first epoch & $20.82 \pm 0.01$ & $21.20 \pm 0.01$ & $21.81 \pm 0.01$ & $23.46 \pm 0.12$ & $24.04 \pm 0.09$ & $24.92 \pm 0.24$ \\
$i$-band second epoch & $20.95 \pm 0.01$ & $21.31 \pm 0.01$ & $21.90 \pm 0.01$ & $23.78 \pm 0.08$ & $23.96 \pm 0.08$ & $24.55 \pm 0.07$ \\
\hline$u$-band second epoch & $22.85 \pm 0.08$ & $23.29 \pm 0.09$ & $24.18 \pm 0.17$ & $>24.58$ & $>24.58$ & $>24.58$ \\
\hline
\end{tabular}

Notes. Uncertainties are quoted at the $1 \sigma$ level. Images D, E, F are not detected in the $u$-band; the $1-\sigma$ detection limit in our data is 24.58 mag. When comparing this photometry to the SDSS photometry reported in Table 2, a correction for the quasar host galaxy should be applied to the SDSS photometry, as detailed in the main text.

brighter images, systematics due to uncertain treatment of the host galaxy are likely dominant, and not addressable with these limited data.

Images $\mathrm{E}$ and $\mathrm{F}$ must be treated with further care. The photometry of image $\mathrm{E}$ is complicated by the blue core of galaxy G3, and image F is simply very faint, and near the core of the brightest cluster galaxy, G1. Particularly at redder wavelengths, this makes its measurement highly uncertain. Furthermore, tests using the full GALFIT model, in which we remeasured noisy realizations of the full model, also show potentially strong biases - up to factors of three-in the photometry of image F. To attempt best possible photometry for these, we proceeded as follows.

First, note that if the colors of the various quasar images are the same, then the relative photometry of residual images in the $g-i$ subtraction in Figure 6 can be used to measure the fainter images. Matched colors are not guaranteed, due both to intrinsic variability (e.g., Schmidt et al. 2012) coupled with time delays, as well as variations induced by differential magnification between the quasar and underlying host galaxy, and possible extinction of individual images due to intervening galaxies in the complex cluster core. However, we can validate the likely variability in color between the images by considering the three brightest and most isolated images $(\mathrm{A}-\mathrm{C})$; these show that the typical scatter about a fixed color is 0.05 in $g-i$ and 0.03 in $g-i$. This uncertainty is much less than the systemic biases noted above in attempts at direct measurement, and so the fixed color assumption is sufficient given these data.

Thus for image $\mathrm{F}$, we use difference images to measure the differential flux of image $\mathrm{F}$ relative to images $\mathrm{A}-\mathrm{C}$. Assuming that $\mathrm{F}$ has the same color as these images, the flux of image $\mathrm{F}$ is then given simply by the mean flux of images A-C times the flux ratio in the difference image. All magnitudes are measured using PSF fitting photometry for consistency. The difference image $g-i$ provides the best measurement of the residual fluxes, and is the basis of the measurements reported in Table 3. As before, we estimate uncertainties by using the fitted GALFIT model to generate and refit noisy realizations of the measured image. In the case of image $\mathrm{F}$, the final reported uncertainty is the scatter in the magnitude offset in the residual image, combined in quadrature with the color scatter between the brighter images.

For image E, we must further account for the residual flux from the core of galaxy G3. We proceed as for image F, but additionally use GALFIT to model a best-fitting Sérsic profile fixed at the center of G3 concurrent with the PSF component for image $\mathrm{E}$.
Table 4

Photometry and Redshift Measurements for Galaxies in the Cluster Core

\begin{tabular}{lcccc}
\hline \hline Galaxy & $i$ & $\begin{array}{c}g-i \\
\left(2^{\prime \prime} 0 \text { Aperture }\right)\end{array}$ & $\begin{array}{c}r-i \\
\left(2^{\prime \prime} \text {.0 Aperture }\right)\end{array}$ & Redshift \\
\hline G1 & 18.74 & 2.71 & 0.97 & $0.493 \pm 0.013$ \\
G2 & 18.97 & 2.53 & 0.96 & $0.496 \pm 0.012$ \\
G3 & 19.37 & 2.45 & 0.93 & $0.489 \pm 0.013$ \\
\hline
\end{tabular}

Finally, we note that each frame is directly calibrated to the SDSS using many hundreds of objects; calibration uncertainties are $0.01 \mathrm{mag}$ at worst.

\subsection{Lensing Cluster}

The maximum separation of the quasar images is $15^{\prime \prime} .1$ (between quasar images $\mathrm{A}$ and $\mathrm{C}$ ) clearly indicative of a clustersized lensing mass. The SDSS photometric redshift estimates of galaxies G1-G3 (see Table 2) are consistent with all three galaxies being located in a cluster at $z \sim 0.5$. As part of the observations described in Sections 3.1 and 3.2 above, all three galaxies were targeted spectroscopically. Redshift estimates were obtained through cross-correlation with the elliptical galaxy template of Kinney et al. (1996), yielding the redshift estimates listed in Table 4. The redshifts are consistent within the measured uncertainties, and all three galaxies G1-G3 clearly belong to the same cluster at a redshift consistent with the SDSS photometric redshift estimates. To further investigate the properties of the lensing cluster, we applied the SExtractor software (Bertin \& Arnouts 1996) for galaxy photometry to the stacked second-epoch MOSCA gri images. Prior to obtaining the photometric measurements, the stacked $r$-and $i$-band images were smoothed to match the resolution of the $g$-band image. Object detection was initially performed on the stacked $g$-band image, and SExtractor was subsequently run in dual-image mode on the stacked images in the other passbands, using the $g$-band image as a reference for object detection. The observed magnitudes were subsequently converted to $\mathrm{AB}$ magnitudes in the SDSS system using objects in the MOSCA field with cataloged SDSS photometry. Total magnitudes were determined using the SExtractor MAG_AUTO parameter, and colors were determined from aperture magnitudes (MAG_APER) within a $2^{\prime \prime}$ diameter aperture. A $g-i$ versus $i$ color-magnitude diagram of non-stellar objects, selected based on their location in a magnitude $-\mu_{\max }$ diagram (where $\mu_{\max }$ is the central surface brightness of objects), is shown in Figure 9. A red sequence is 


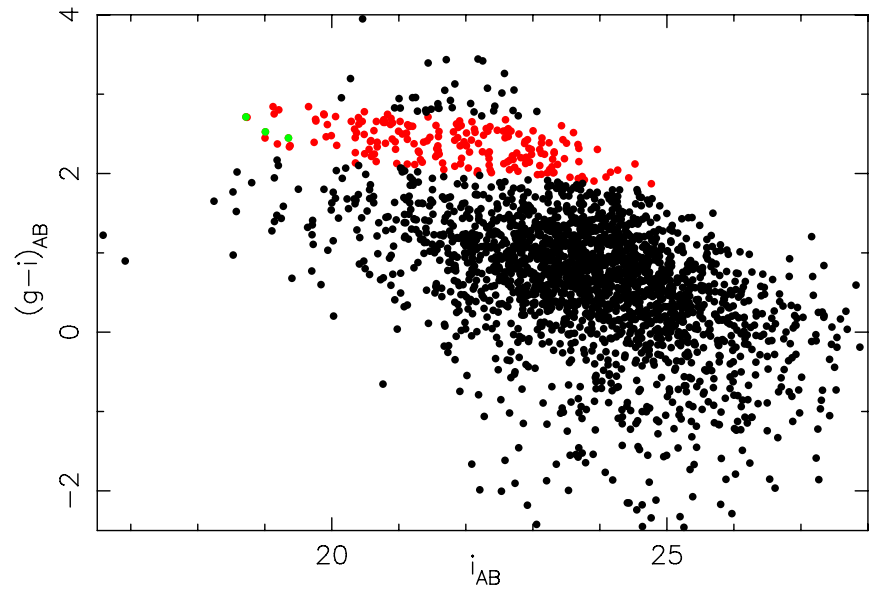

Figure 9. Color-magnitude diagram of 3033 non-stellar objects detected in the MOSCA field containing SDSS J2222+2745. The galaxies G1-3 are indicated by green dots. Other galaxies with $g-i$ colors within \pm 0.35 mag from the red sequence formed by early-type galaxies in the lensing cluster are indicated by red dots.

(A color version of this figure is available in the online journal.)

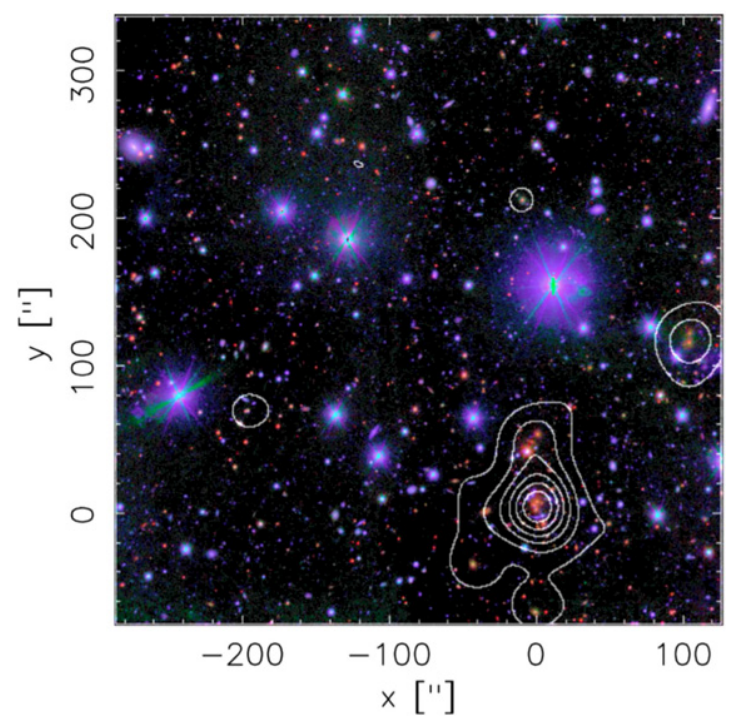

Figure 10. Contour plot showing a smoothed map of the light distribution of the red sequence galaxies indicated by the red and green colors in Figure 9. The background figure is a gri composite based on the second-epoch MOSCA images. The coordinates are relative to the position of galaxy $\mathrm{G} 1$, which closely coincides with the cluster lens (see Section 4). A strong peak in the light distribution is seen at this location.

(A color version of this figure is available in the online journal.)

evident at $g-i \sim 2.5$, similar to the $g-i$ colors of the three galaxies G1-G3 (see Table 4). Figure 10 shows a smoothed map of the light distribution of red sequence galaxies over the observed MOSCA field. A strong peak in the luminosity distribution is located at the position of galaxies G1-G3.

\subsection{Giant Arc}

The identification of SDSS J2222+2745 as a candidate gravitational lens system was partially due to recognition of the giant arc just south of the cluster center. The extent of the arc in our deepest stacked $g$-band image (see Figure 3 ) is $10^{\prime \prime}$. Our SExtractor photometry (see Section 3.4) of the arc yields $g=21.57$, and $u-g=0.87,-g-r=0.21, g-i=0.41$, where the colors are measured in $2^{\prime \prime}$ diameter apertures centered on the brightest central portion of the arc. We note that the quoted magnitude of the arc can be considered a lower limit on its brightness, given the very extended morphology of the arc. Although SExtractor attempts to treat the arc and the WD as separate objects, a more careful modeling of the shape of the arc (including PSF modeling and removal of the superposed WD point source) would be required to obtain an accurate total magnitude. The spectrum of the arc shows a single emission line at $\lambda_{\text {obs }}=\sim 4015 \AA$, which we interpret as Ly $\alpha$ at $z=2.30$. The alternative interpretation of [O II] (3727 $\AA$ ) at $z=0.077$ would put the arc in front of the cluster, which is obviously inconsistent with the lensed morphology of the arc. The lens model presented in the following section does not predict a counter-image of the arc.

\section{LENS MODEL}

We compute a parametric lens model using the publicly available software Lenstool (Jullo et al. 2007), through Markov chain Monte Carlo (MCMC) minimization in the image plane. The lens is modeled with several components: a cluster halo, brightest cluster galaxy (BCG), and cluster galaxies. All the components are represented by a pseudo-isothermal elliptical mass distributions (PIEMD; ${ }^{11}$ Limousin et al. 2005; Elíasdóttir et al. 2007), parameterized by its position $x, y$; a fiducial velocity dispersion $\sigma$; a core radius $r_{\text {core }}$; a scale radius $r_{s}$; ellipticity $e=\left(a^{2}-b^{2}\right) /\left(a^{2}+b^{2}\right)$, where $a$ and $b$ are the semi major and semi minor axes, respectively; and a position angle $\theta$. All the parameters of the cluster halo distribution are allowed to vary within priors, except for $r_{s}$, which is not constrained by the lensed images.

Cluster members are selected based on their $g-i$ color in a color-magnitude diagram, with respect to the cluster red sequence (see Figure 9), and their position, ellipticity, and position angle are fixed at their observed values, as measured with SExtractor. The parameters $\sigma$ and $r_{s}$ are determined through scaling relations (see Limousin et al. 2005 for a description of the scaling relations). Three galaxies, G1, G2, and G3, are in close proximity to three lensed images of the quasar, and thus affect their positions more directly than other cluster members. To account for the intrinsic scatter in the scaling relations, we allow some of the parameters of these three galaxies to be modeled separately from the rest of the cluster galaxies.

As constraints, we use the redshift and positions of the images of the quasar and of the lensed galaxy at $z=2.30$. We have a total of 12 free parameters: 6 free parameters for the cluster halo, and 2 free parameters for each of the galaxies, and 14 constraints. The best-fit parameters are listed in Table 5. The uncertainties were estimated through the MCMC sampling of the parameter space. For parameters that were not well constrained, we list the priors that were set in the minimization. Caustics, critical lines, and image positions derived from the best fit model are shown in Figure 11. To account for systematics due to misidentification of the sixth image, we also compute a model assuming only five images. The two families of models are similar, although we note that G1 is poorly constrained in the model without image F.

The image-plane rms scatter of the quasar images is $00^{\prime \prime} 14$ in the five-image model and 0.18 in the six-image model; the rms of the blue arc is somewhat larger $\left(0^{\prime \prime} .26,0^{\prime \prime} 38\right.$, respectively), due to the difficulty of measuring its positional constraints, and the larger weight given to the quasar images in the modeling process.

\footnotetext{
11 This profile is formally the same as dual pseudo-isothermal elliptical mass
} distribution (see Elíasdóttir et al. 2007). 
Table 5

Best-fit Lens Model Parameters

\begin{tabular}{|c|c|c|c|c|c|c|c|}
\hline $\begin{array}{l}\text { Halo } \\
\text { (PIEMD) }\end{array}$ & $\begin{array}{l}\text { R.A. } \\
\left({ }^{\prime \prime}\right)\end{array}$ & $\begin{array}{c}\text { Decl. } \\
\left({ }^{\prime \prime}\right)\end{array}$ & $e$ & $\begin{array}{c}\theta \\
(\operatorname{deg})\end{array}$ & $\begin{array}{l}r_{\text {core }} \\
(\mathrm{kpc})\end{array}$ & $\begin{array}{c}r_{\mathrm{s}} \\
(\mathrm{kpc})\end{array}$ & $\begin{array}{c}\sigma_{0} \\
\left(\mathrm{~km} \mathrm{~s}^{-1}\right)\end{array}$ \\
\hline \multicolumn{8}{|c|}{ Model 1: constraints from 5 QSO images. rms $\mathrm{QSO}=00^{\prime \prime} 14$} \\
\hline Cluster & $-0.14_{-1.03}^{+0.50}$ & $1.18_{-0.81}^{+1.90}$ & $0.66_{-0.14}^{+0.25}$ & $97_{-4}^{+5}$ & $26_{-42}^{+15}$ & [2000] & $580_{-163}^{+64}$ \\
\hline G1 & {$[0.0]$} & {$[0.0]$} & {$[0.26]$} & {$[3.8]$} & $0.77_{-0.23}^{+0.77}$ & [47] & $155_{-168}^{+55}$ \\
\hline $\mathrm{G} 2$ & {$[-0.596]$} & {$[-5.220]$} & {$[0.21]$} & {$[75.5]$} & $0.13\left[\begin{array}{ll}0.0 & 0.3\end{array}\right]$ & {$[24]$} & $212_{-44}^{+40}$ \\
\hline $\mathrm{L}^{*}$ galaxy & $\cdots$ & $\cdots$ & $\cdots$ & $\cdots$ & {$[0.15]$} & {$[20]$} & [100] \\
\hline \multicolumn{8}{|c|}{ Model 2: constraints from 6 QSO images. $\mathrm{rms}_{\mathrm{QSO}}=0^{\prime \prime} 18$} \\
\hline Cluster & $-0.16_{-0.71}^{+0.58}$ & $2.10_{-0.90}^{+2.58}$ & $0.75_{-0.05}^{+0.13}$ & $96_{-3}^{+4}$ & $37_{-40}^{+13}$ & [2000] & $578_{-114}^{+50}$ \\
\hline G1 & {$[0.0]$} & {$[0.0]$} & {$[0.26]$} & [3.8] & $0.05_{-0.93}^{+0.04}$ & [47] & $303_{-46}^{+34}$ \\
\hline $\mathrm{G} 2$ & {$[-0.596]$} & {$[-5.220]$} & {$[0.21]$} & [75.5] & $0.15\left[\begin{array}{lll}0.0 & 0.3\end{array}\right]$ & [24] & $226_{-36}^{+29}$ \\
\hline G3 & {$[-1.762]$} & {$[-2.308]$} & {$[0.41]$} & {$[-18.3]$} & $0.15\left[\begin{array}{lll}0.0 & 0.3\end{array}\right]$ & {$[20]$} & $235_{-33}^{+46}$ \\
\hline
\end{tabular}

Notes. All coordinates are measured in arcseconds relative to the center of G1, at [R.A., decl.] $=[335.53573,27.75986]$. The ellipticity is expressed as $e=\left(a^{2}-b^{2}\right) /\left(a^{2}+b^{2}\right) . \theta$ is measured north of west. Error bars correspond to $1 \sigma$ confidence level as inferred from the MCMC optimization. Values in square brackets are for parameters that are not optimized. Errors in square brackets represent the lower and upper limits that were set as prior in the optimization process, for parameters that were not well constrained by the data. The location and the ellipticity of the matter clumps associated with the cluster galaxies were kept fixed according to their light distribution.

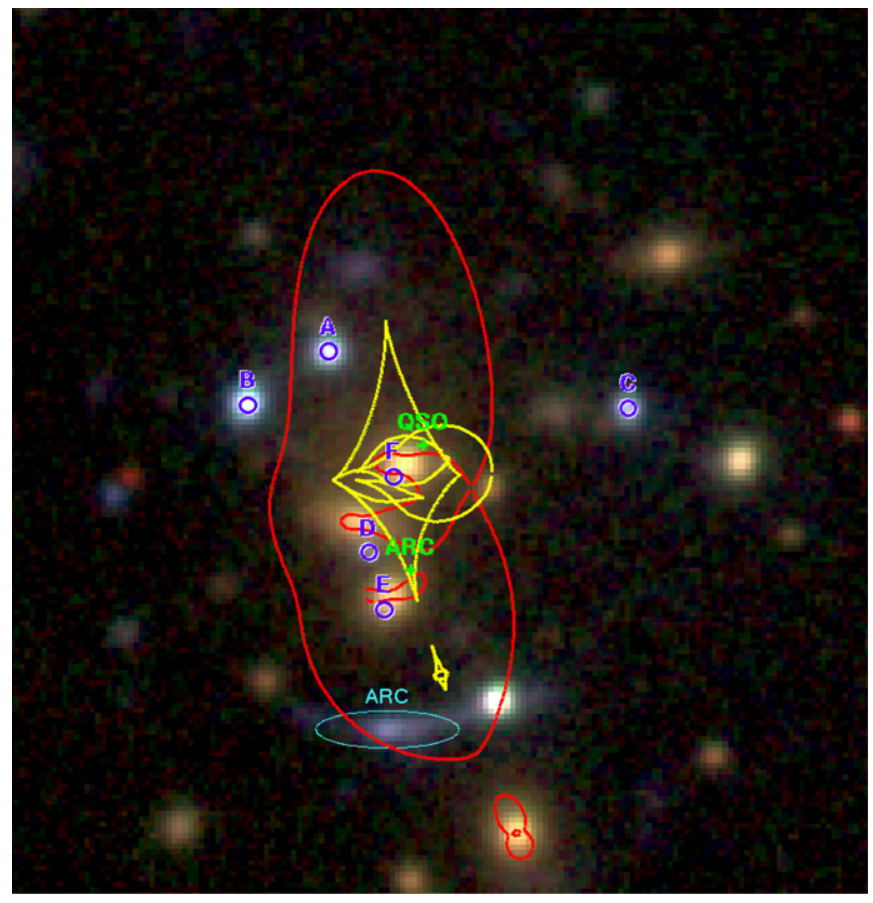

Figure 11. Caustics, critical lines, and image positions from the best-fit model, superposed on a gri color image of the SDSS J2222+2745 lens system. The critical curves for a source at the quasar redshift $(z=2.82)$ are indicated in red. The caustic curves in the source plane are shown as yellow lines, and the predicted source locations of the quasar and arc with respect to these curves are indicated by green crosses. The observed locations of the six quasar images $\mathrm{A}-\mathrm{F}$ are indicated by blue circles.

(A color version of this figure is available in the online journal.)

Both models successfully predict the locations of all the observed images (either five or six, depending on the model constraints), and do not predict additional images, with the exception of demagnified central images that cannot be detected in our data: the model with six images predicts a demagnified seventh image close to the center of galaxy G2. This is expected in this strong-lensing configuration. Our imaging data do not reveal any hint of this seventh image, though a positive iden- tification might be possible with the superior spatial resolution of Hubble Space Telescope (HST). A careful re-examination of the spectroscopic data for G2 shown in Figure 4 and plotted in Figure 5 may indicate a weak signature of the Ly $\alpha$ line from the seventh image $(\mathrm{G})$, but this feature is almost at the level of the noise, and more sensitive spectroscopy with $8-10 \mathrm{~m}$ class telescopes will be required for a definite detection.

Table 6 lists the model-predicted magnifications and time delays for the two model families. Uncertainties are computed from a suite of models, drawn from the MCMC chain, that represent the $1 \sigma$ uncertainty in the parameter space. All time delays are given relative to image $\mathrm{A}$. The best-fit models predict short time delays of order of 100 days between the close pair (A-B), and between the central images (D-E-F). Image C precedes A and B by 1400 days, and D-E-F follow A and B by $700-1000$ days. In the six-image model, $66 \%$ of the models, as well as the best-fit model, predict the order of the images to be $\mathrm{C}-\mathrm{B}-\mathrm{A}-\mathrm{F}-\mathrm{E}-\mathrm{D}$. All the models predict that image $\mathrm{C}$ is the first one to occur, followed by $\mathrm{B}-\mathrm{A}$ (in $93 \%$ of the models) or A-B (7\%); followed by F-E-D (72\%) F-D-E (16\%) or D-F-E $(12 \%)$. In the five-image model, $62 \%$ of the models, as well as the best-fit model, predict $\mathrm{C}-\mathrm{B}-\mathrm{A}-\mathrm{E}-\mathrm{D}$; here too, all the models predict that $\mathrm{C}$ is the first image to occur, followed by $\mathrm{B}-\mathrm{A}(77 \%)$ or A-B (23\%), and then E-D (81\%) or D-E (19\%).

For easy comparison between the predicted and observed relative fluxes, Table 6 lists relative fluxes calculated from the second-epoch $g$-band photometry (which has the smallest measurement uncertainties) in Table 3 , scaled such that the $\mathrm{B}$-image flux matches the predicted magnification of the $\mathrm{B}$ image. Even without taking into account additional uncertainties related to intrinsic variability in the quasar coupled with the fact that the time delays have not yet been measured, the predicted and observed flux ratios are found to be consistent within the errors. Hence, there is at present no evidence for anomalous flux ratios caused by, e.g., microlensing or lens substructure close to the line of sight of the quasar images.

The effective Einstein radius for a source at the quasar redshift $(z=2.82)$ is defined as $\theta_{\mathrm{E}, \text { eff }} \equiv \sqrt{A / \pi}$, where $A$ is the area enclosed by the tangential critical curve for that redshift (Gralla 
Table 6

Model-predicted Magnifications and Time Delays and Observed Relative Fluxes

\begin{tabular}{|c|c|c|c|c|c|}
\hline Image & Magnification (1) & $\begin{array}{c}\text { Time Delay (1) } \\
\text { (days) }\end{array}$ & Magnification (2) & $\begin{array}{c}\text { Time Delay (2) } \\
\text { (days) }\end{array}$ & Relative Flux \\
\hline A & $5.7_{-3.1}^{+14.5}$ & 0 & $4.7_{-1.6}^{+3.4}$ & 0 & $7.94 \pm 0.11$ \\
\hline $\mathrm{C}$ & $2.5_{-0.6}^{+3.6}$ & $-1399_{-776}^{+850}$ & $2.4_{-0.3}^{+1.1}$ & $-1368_{-344}^{+456}$ & $2.94 \pm 0.04$ \\
\hline $\mathrm{D}$ & $0.6_{-0.2}^{+0.7}$ & $960_{-254}^{+365}$ & $0.7_{-0.2}^{+0.3}$ & $931_{-233}^{+237}$ & $0.73 \pm 0.02$ \\
\hline $\mathrm{E}$ & $0.7_{-0.3}^{+0.9}$ & $851_{-278}^{+448}$ & $0.6_{-0.2}^{+0.3}$ & $806_{-224}^{+370}$ & $0.47 \pm 0.04$ \\
\hline $\mathrm{F}$ & $\ldots$ & $\ldots$ & $0.5_{-0.2}^{+0.5}$ & $723_{-161}^{+223}$ & $0.27 \pm 0.02$ \\
\hline
\end{tabular}

Notes. Model 1: constraints from five QSO images. Model 2: constraints from six QSO images. Time delay is given in days, relative to image A. The relative flux values listed here are calculated from the observed second-epoch $g$-band magnitudes listed in Table 3 , scaled to match the predicted magnification of image B in model 2.

et al. 2011). For the best-fit models (with five or six quasar images), $R_{\mathrm{E} \text {,eff }}=7$ ".34, which corresponds to $44.8 \mathrm{kpc}$. The total projected mass at the core of the cluster, based on the stronglensing model, is best constrained inside the critical curve. The total projected masses $M(<r)$ in cylindrical apertures of radii $r$, are $M\left(<R_{\mathrm{E}, \mathrm{eff}}\right)=11.2_{-0.4}^{+0.3} \times 10^{12} M_{\odot}, M(<100 \mathrm{kpc})=27_{-2}^{+4} \times$ $10^{12} M_{\odot}$, and $M(<200 \mathrm{kpc})=56_{-7}^{+16} \times 10^{12} M_{\odot}$ for the siximage lens model. The corresponding values for the five-image model are $M\left(<R_{\mathrm{E}, \mathrm{eff}}\right)=11.3_{-0.5}^{+0.3} \times 10^{12} M_{\odot}, M(<100 \mathrm{kpc})=$ $29_{-4}^{+8} \times 10^{12} M_{\odot}$, and $M(<200 \mathrm{kpc})=59_{-12}^{+28} \times 10^{12} M_{\odot}$.

\section{SUMMARY AND DISCUSSION}

\subsection{Previous Observations and Predictions of High Image Multiplicities}

We have identified SDSS J2222+2745 as a gravitational lens system producing six observed images of the same quasar. This is the third known cluster-scale lens and the only such lens producing more than five observed images. The only previously known six-image active galactic nucleus lens is B1359+154 (Rusin et al. 2001), where a radio source at $z_{s}=3.235$ is being lensed by a compact group of three galaxies at $z_{l} \sim 1$. However, the quasar image separations we observe in SDSS J2222+2745 are an order of magnitude larger than in B1359+154, and the lensed images are $\sim 3$ mag brighter at optical wavelengths, making six images detectable in ground-based imaging of SDSS J2222+2745. The B1359+154 lensed image configuration is similar to what we find in SDSS J2222+2745, with the three fainter images located in close proximity to the three galaxies situated within the critical curves of the lens system, and three brighter images located further away from these galaxies. However, the lens model of B1359+154 presented by Rusin et al. (2001) does not allow a significant dark matter halo associated with the galaxy group in addition to the three halos corresponding to the three individual galaxies. In contrast, in our model the cluster halo component is the dominant contributor to the observed image splitting of SDSS J2222+2745. Specifically, the cluster halo comprises $61 \%$ of the total enclosed mass within $R_{\mathrm{E}, \text { eff }}, 78 \%$ of the mass within $100 \mathrm{kpc}$, and $87 \%$ of the mass within $200 \mathrm{kpc}$. However, as with any parametric multicomponent cluster mass model, these numbers will be sensitive to the chosen mass profile parameterization for the different components.

Since cluster-scale quasar lenses and image multiplicities $>5$ are both rare, we would expect a lensing system such as SDSS J2222+2745 to be extremely rare. There are no appropri- ate quantitative predictions for the occurrence of this type of lens systems available in the literature. Evans \& Witt (2001) calculate the incidence of sextuplet and octuplet image systems (with demagnified seventh and ninth images, respectively) and estimate that these constitute $\sim 1 \%$ of the population of quasars multiply imaged by galaxy-scale lenses. This is consistent with the single known sextuplet case of B1359+154 among the $\sim 100$ known galaxy-scale quasar lenses. For the larger image separations produced by cluster lenses, published studies of the probabilities of different lensing multiplicities (Oguri \& Keeton 2004; Li et al. 2007; Minor \& Kaplinghat 2008) only include double, quadruple (with demagnified third and fifth images, respectively) and naked-cusp configurations. Li et al. (2007) state that they do not expect a significant fraction of sources to have image numbers exceeding 5 .

\subsection{Comparing the Lens and Source Redshifts to Predictions}

Both the quasar source redshift and cluster lens redshift of SDSS J2222+2745 are comparable to the two other cluster quasar lenses and in good agreement with theoretical expectations: the observed lens redshift in SDSS J2222+2745 is $z=0.49$, which is similar to, but slightly lower than, the cluster lenses in SDSS J1004+4112 $(z=0.68)$ and SDSS J1029+2623 $(z=0.58)$. This redshift is very close to the peak (at $z \simeq 0.5$ ) of the predicted lens redshift distribution for photometrically selected wide-separation quasars in the SDSS (Li et al. 2007). The observed quasar source redshift in SDSS J2222+2745 is $z=2.82$, slightly higher than the sources in SDSS J1004+4112 $(z=1.73)$ and SDSS J1029+2623 $(z=2.20)$. These three redshifts are all near the peak (around $z=2$ ) of the predicted source redshift distribution for photometrically selected wideseparation quasars in the SDSS (Li et al. 2007).

\subsection{Prospects for Time Delay Measurements}

The photometry reported in Section 3.3 shows significant variability in the quasar, demonstrating the feasibility of making time delay measurements in SDSS J2222+2745 from monitoring campaigns using ground-based 2-4 m class telescopes. We have recently initiated a monitoring program for SDSS J2222+2745 at the NOT for this purpose. The predicted time delays from our lens model (see Section 4) indicate that three independent time delays $\Delta t_{\mathrm{AB}}\left(\equiv \Delta t_{B}-\Delta t_{A}\right), \Delta t_{\mathrm{ED}}$ and $\Delta t_{\mathrm{FE}}$ in the system are all of the order of 100 days. This suggests that several time delay measurements could, in the best case, be obtained from a single season of monitoring. The predicted time delays between image $\mathrm{C}$ and the close pair $(\mathrm{A}-\mathrm{B})$ 
and between $(\mathrm{A}-\mathrm{B})$ and the central images $(\mathrm{D}-\mathrm{E}-\mathrm{F})$ are of the order of 1400 days and 700-1000 days, respectively. These values are similar to the two longest predicted time delays for SDSS J1004+4112, of 1218 and 1674 days, respectively (Oguri 2010) and the measured longest time delay in SDSS J1029+2623 of $\Delta t_{\mathrm{AB}}=(744 \pm 10)$ days (Fohlmeister et al. 2013).

\subsection{Future Work}

Morphological, photometric, and spectroscopic evidence presented in this paper strongly suggest the presence of a sixth (F) image of the quasar located closest to the lensing mass center. The odd number theorem of strong gravitational lensing theory (McKenzie 1985; Petters \& Werner 2010) will then require the presence of a seventh $(G)$ image, for which our model predicts a location close to the center of cluster galaxy G2. In the case of galaxy-scale lenses, the faintest image of a multiply lensed quasar is typically highly demagnified and hence unobservable in the vast majority of cases. However, the shallower central slopes of the mass density profiles of clusters increase the chance of observing the final odd image, as exemplified by the identification of the demagnified fifth central image in SDSS J1004+4112 using HST imaging (Inada et al. 2005, 2008).

While the data presented in this paper do not provide conclusive evidence for the presence of the seventh image, there is a hint of Ly $\alpha$ emission from this image in the spectrum of G2. If this feature is indeed emission from the quasar, a firm confirmation would be possible through spectroscopic observations with 8-10 m class telescopes.

While time delay measurements would provide important additional modeling constraints on the SDSS J2222+2745 lens system, follow-up HST imaging will also be crucial to improve the modeling constraints from the observed locations of the quasar images and hopefully provide morphological and photometric confirmation of the predicted seventh $(\mathrm{G})$ image close to the center of galaxy G2. Further constraints on the lens system could also come from spatially resolved, multiply imaged structures within the giant arc and from the identification of additional fainter, multiply imaged, galaxies as revealed by the HST observations of SDSS J1004+4112 (Sharon et al. 2005) and SDSS J1029+2623 (Oguri et al. 2013).

\subsection{Broader Significance of This Discovery}

The significance of the discovery of SDSS J2222+2745 is twofold. First, the presence of six quasar images (and potential future confirmation of a seventh image), spread over a range of clustercentric radii, with good prospects for future time delay measurements, coupled with the presence of an additional lensed source producing a bright giant arc, can provide unique detailed measurements of the matter distribution in the core of a cluster. Such measurements provide a stringent test of the scenario of structure formation, including the properties of dark matter in cluster cores.

Second, adding our new discovery of SDSS J2222+2745 to the previous detections of SDSS J1004+4112 and SDSS J1029+2623 makes a significant contribution toward establishing quasars strongly lensed by galaxy clusters as a cosmological tool. The probability of such rare lenses depends very sensitively on cosmological parameters, in particular the normalization of the matter power spectrum $\sigma_{8}$ and the matter density $\Omega_{M}$ (e.g., Li et al. 2007). Even though no directly applicable predictions can be found in the literature, the occurrence of lens systems with image separations and image multiplicities as high or higher than SDSS J2222+2745 must necessarily be exceptionally rare, and this could conceivably be the only such system, provided that the current $\Lambda \mathrm{CDM}$ paradigm is correct.

H.D. acknowledges support from the Research Council of Norway. M.D.G. thanks the Research Corporation for support of this work through a Cottrell Scholars award. K.S. acknowledges support from the University of Michigan's President's Postdoctoral Fellowship. J.P.U.F. acknowledges support from the ERC-StG grant EGGS-278202. The Dark Cosmology Centre is funded by the DNRF. We thank the NOT staff, in particular our support astronomer Stefan Geier, for excellent support, including accommodating a last-minute instrument change which allowed the spectroscopic confirmation of the D image and discovery of the E image of the quasar.

\section{REFERENCES}

Aihara, H., Allende Prieto, C., An, D., et al. 2011, ApJS, 193, 29

Bayliss, M. B., Hennawi, J. F., Gladders, M. D., et al. 2011, ApJS, 193, 8 Bertin, E., \& Arnouts, S. 1996, A\&AS, 117, 393

Elíasdóttir, Á., Limousin, M., Richard, J., et al. 2007, arXiv:0710.5636

Evans, N. W., \& Witt, H. J. 2001, MNRAS, 327, 1260

Fohlmeister, J., Kochanek, C. S., Falco, E. E., et al. 2013, ApJ, 764, 186

Fohlmeister, J., Kochanek, C. S., Falco, E. E., Morgan, C. W., \& Wambsganss, J. 2008, ApJ, 676, 761

Gralla, M. B., Sharon, K., Gladders, M. D., et al. 2011, ApJ, 737, 74

Hennawi, J. F., Dalal, N., \& Bode, P. 2007, ApJ, 654, 93

Hennawi, J. F., Gladders, M. D., Oguri, M., et al. 2008, AJ, 135, 664

Inada, N., Oguri, M., Falco, E. E., et al. 2008, PASJ, 60, L27

Inada, N., Oguri, M., Keeton, C. R., et al. 2005, PASJ, 57, L7

Inada, N., Oguri, M., Morokuma, T., et al. 2006, ApJL, 653, L97

Inada, N., Oguri, M., Pindor, B., et al. 2003, Natur, 426, 810

Jullo, E., Kneib, J.-P., Limousin, M., et al. 2007, NJPh, 9, 447

Kinney, A. L., Calzetti, D., Bohlin, R. C., et al. 1996, ApJ, 467, 38

Koester, D., Girven, J., Gänsicke, B. T., \& Dufour, P. 2011, A\&A, 530, A114

Lewis, G. F., Carilli, C., Papadopoulos, P., \& Ivison, R. J. 2002, MNRAS, 330, L15

Li, G. L., Mao, S., Jing, Y. P., Lin, W. P., \& Oguri, M. 2007, MNRAS, 378,469

Limousin, M., Kneib, J.-P., \& Natarajan, P. 2005, MNRAS, 356, 309

McKenzie, R. H. 1985, JMP, 26, 1592

Minor, Q. E., \& Kaplinghat, M. 2008, MNRAS, 391, 653

Oguri, M. 2010, PASJ, 62, 1017

Oguri, M., \& Keeton, C. R. 2004, ApJ, 610, 663

Oguri, M., Ofek, E. O., Inada, N., et al. 2008, ApJL, 676, L1

Oguri, M., Schrabback, T., Jullo, E., et al. 2013, MNRAS, 429, 482

Peng, C. Y., Ho, L. C., Impey, C. D., \& Rix, H.-W. 2010, AJ, 139, 2097

Petters, A. O., \& Werner, M. C. 2010, GReGr, 42, 2011

Refsdal, S. 1964, MNRAS, 128, 307

Refsdal, S. 2004, in Thinking, Observing and Mining the Universe, ed. G. Miele \& G. Longo (Italy: Univ. Napoli "Federico II"), 231

Rusin, D., Kochanek, C. S., Norbury, M., et al. 2001, ApJ, 557, 594

Schmidt, K. B., Rix, H.-W., Shields, J. C., et al. 2012, ApJ, 744, 147

Sharon, K., Ofek, E. O., Smith, G. P., et al. 2005, ApJL, 629, L73

Vanden Berk, D. E., Richards, G. T., Bauer, A., et al. 2001, AJ, 122, 549

York, D. G., Adelman, J., Anderson, J. E., Jr., et al. 2000, AJ, 120, 1579 